OPEN ACCESS

Edited by:

Mamoru Ayusawa,

Nihon University Itabashi

Hospital, Japan

Reviewed by:

Toni Hospach,

Klinikum Stuttgart, Germany

Riccardo Castagnoli,

University of Pavia, Italy

${ }^{*}$ Correspondence:

Yusuke Okubo

sunning_dale@yahoo.co.jp

Specialty section:

This article was submitted to

Pediatric Immunology,

a section of the journal

Frontiers in Pediatrics

Received: 16 January 2020 Accepted: 05 March 2020 Published: 24 March 2020

Citation:

Okubo Y, Miura M, Kobayashi T, Morisaki N, Michihata N, Matsui H, Fushimi $\mathrm{K}$ and Yasunaga $\mathrm{H}$ (2020) The

Impact of Changes in Clinical

Guideline on Practice Patterns and Healthcare Utilizations for Kawasak

Disease in Japan.

Front. Pediatr. 8:114

doi: 10.3389/fped.2020.00114

\section{The Impact of Changes in Clinical Guideline on Practice Patterns and Healthcare Utilizations for Kawasaki Disease in Japan}

\author{
Yusuke Okubo 1,2,3*, Masaru Miura ${ }^{4}$, Tohru Kobayashi ${ }^{5}$, Naho Morisaki ${ }^{2}$, \\ Nobuaki Michihata ${ }^{3}$, Hiroki Matsui ${ }^{3}$, Kiyohide Fushimi ${ }^{6}$ and Hideo Yasunaga ${ }^{3}$ \\ ${ }^{1}$ Department of Epidemiology, UCLA Fielding School of Public Health, Los Angeles, CA, United States, ${ }^{2}$ Department of \\ Social Medicine, National Center for Child Health and Development, Tokyo, Japan, ${ }^{3}$ Department of Clinical Epidemiology and \\ Health Economics, School of Public Health, The University of Tokyo, Tokyo, Japan, ${ }^{4}$ Department of Cardiology, Tokyo \\ Metropolitan Children's Medical Center, Tokyo, Japan, ${ }^{5}$ Division of Clinical Research Planning, Department of Development \\ Strategy, Center for Clinical Research and Development, National Center for Child Health and Development, Tokyo, Japan, \\ ${ }^{6}$ Department of Health Policy and Informatics, Tokyo Medical and Dental University Graduate School of Medicine, Tokyo, \\ Japan
}

Objective: Previous studies showed the efficacy of glucocorticoids on prevention of coronary artery lesions (CAL) among Kawasaki disease (KD) patients, and clinical guideline for KD in Japan was changed regarding glucocorticoid use in 2012. However, little is known regarding how the guideline change had impacts on healthcare utilizations and clinical outcomes.

Methods: We conducted a retrospective observational study using national inpatient database in Japan among KD patients aged under 18 years during 2010-2015. Recent trends in practice patterns were analyzed, and we divided the hospitals into four groups based on glucocorticoid use: (1) consistently using hospital, (2) started using hospital, (3) stopped using hospital, and (4) never using hospital. Then, we compared healthcare utilizations and risks of coronary artery lesions before and after the guideline change.

Results: We identified 24,517 inpatients with KD. From 2010 to 2014, use of glucocorticoid increased from 8.9 to $17.4 \%$ of $\mathrm{KD}$ inpatients. All types of hospitals showed reduction in coronary artery lesions, but the reduction was the most prominent in hospitals that started using glucocorticoid therapy after clinical guideline change in 2012 (adjusted OR, 0.22; 95\% Cl, 0.07-0.68). Also, Glucocorticoid consistently using hospitals, started using hospitals, and never using hospitals showed reductions in hospitalization costs, whereas hospitals that stopped using glucocorticoids after clinical guideline change had elevated healthcare costs as opposed to natural trends observed in other groups. Guideline complying hospitals had the greatest reductions in healthcare costs.

Conclusions: The early stage glucocorticoid use could be a cost-saving strategy for treatment for KD patients without increasing risks of $\mathrm{CAL}$.

Keywords: coronary artery aneurysm, Kawasaki disease, Diagnosis Procedure Combination inpatient database, healthcare utilization, clinical guideline, practice pattern 


\section{INTRODUCTION}

Kawasaki disease (KD) is an acute systemic vasculitis among children (1-5). KD is one of the most common cause of acquired heart disease during childhood in developed countries, and the devastating sequelae are coronary artery lesions (CAL) (1-5). An early suppression of vessel inflammation is thought to be important to prevent development of CAL (1-5).

The standard initial treatments are high-dose intravenous immune-globulin (IVIG) and aspirin (ASA) for children with $\mathrm{KD}$ in the acute phase $(2,5-7)$. Most of the previous studies indicated that $15-20 \%$ of KD patients failed to respond to the initial IVIG and ASA treatments, and the non-responders were more likely to develop CAL than IVIG responders, indicating the further requirement for alternative treatment strategies (814). In Japan, randomized controlled studies were conducted, which showed the effectiveness of corticosteroids added to IVIG and ASA in acute phase among only high-risk patients using the scoring systems $(15,16)$. Based on these results, the 2012 Clinical Guideline for Medical Treatment of Acute Stage Kawasaki Disease in Japan describes recommendation for adding corticosteroid to the IVIG and ASA in acute phase to potential high-risk patients or non-responders by the clinical scoring systems (2). Thus, currently many pediatricians in Japan are expected to apply early initial anti-inflammatory therapy (glucocorticoids to the conventional treatment) for KD patients with high clinical scores (4). However, no previous study has investigated the effects of change in the guideline on the trends in clinical practice patterns and healthcare utilizations at a national level.

Therefore, our study investigated the recent changes in clinical practice patterns and healthcare utilizations among children hospitalized with $\mathrm{KD}$, using a national inpatient database in Japan. Furthermore, we also ascertained how the change in KD guidelines impacted on health care costs and risks of CAL among $\mathrm{KD}$ patients at hospital levels.

\section{METHODS}

\section{Study Population and Participation}

We extracted hospital discharge records of children under 18 years of age hospitalized with KD using the Diagnosis Procedure Combination (DPC) database, a national inpatient database, between July 1, 2010 and March 31, 2015. The details of the DPC database have been described elsewhere (17). In short, the data in the DPC was obtained from more than 1,000 hospitals, and it covers $\sim 55 \%$ of data among all inpatients who were admitted to acute-care hospitals in Japan. The DPC data has hospital discharge records and administrative claims information for about seven million inpatients per year. The database contains information on the following: the patient's primary diagnosis; pre-existing comorbidities on admission; complications and comorbidities during hospitalization; the patients' demographic information such as age, sex, and body weight; procedures and treatments. We imputed missing values of body weight based on ideal body weight as defined by WHO recommendations (18). We obtained the approval for the present study from the
Institutional Review Board at The University of Tokyo. The Board waived the requirement for informed consent because of the anonymous nature of the data.

Hospitalizations with $\mathrm{KD}$ were identified using the International Classification of Diseases, Tenth Revision code (ICD-10 code, M30.3) as the main diagnosis at admission. We included KD patients who were aged $<18$ years and those who were treated with initial IVIG for their first-time admissions in order to maximize the diagnostic accuracy and prevent misclassifications of KD.

\section{Measurements of Variables}

Patient demographic consisted of age, sex, weight in $\mathrm{kg}$, month and year of admission, ambulance use, types of hospitals (academic or non-academic), dose of IVIG, use of glucocorticoids, cyclosporine A, infliximab, ulinastatin, plasmapheresis, and the need for intensive care.

The outcomes of interest were total hospitalization and drug costs, development of CAL, readmission, total length of hospital stay in days, total IVIG dose, and additional treatment. Development of CAL was identified using an ICD-10 code (I25.4: aneurysm of coronary vessels) and use of antithrombotic drugs (warfarin, ticlopidine, and clopidogrel), assuming that the ICD10 code reflected all diagnoses of CAL and that antithrombotic use was for medium- or large-sized CAL.

\section{Statistical Analyses}

We investigated the yearly trends in treatment patterns, risk of CAL and readmission, total hospitalization costs, and length of hospital stay (LOS) from fiscal year 2010 to 2014. To evaluate the trends, we constructed multivariable mixed effects logistic and linear regression models that had fiscal year of admission as an independent categorical variable. All models included fixed effects for patient characteristics and random effects for hospitals to account for clustering.

To capture the secular trends in glucocorticoid use within 2 days of initiation of IVIG for Kawasaki disease, we calculated rate of glucocorticoid use in the initial phase. The rates were calculated based on the number of patients who received glucocorticoids in the initial phase as the numerator and the number of patients who were hospitalized with $\mathrm{KD}$ as the denominator. A Poisson regression model was used with the natural $\log$ of the number of hospitalized patients with $\mathrm{KD}$ by months as an offset, and the scale parameter was added to the model to allow the variance to be proportional rather than equal to the mean to account for the overdispersion (19). We applied a log-linear spline regression model because we assumed that the rate of glucocorticoid use in the initial phase started increasing on January 2012 (3 months before the first randomized controlled study for the effects of glucocorticoid on prevention of CAL was published) and leveled off after March 2013 (3 months after the change in KD guideline).

To investigate the impacts of change in clinical practice guideline on risks of CAL and hospitalization costs at hospital levels, we divided the hospitals into four groups based on glucocorticoid use within 2 days of initiation of IVIG. As 
glucocorticoid use increased from January 2012 to March 2013 (Figure 1), we considered hospitals that used glucocorticoids during the acute phase of $\mathrm{KD}$ before and after the period as consistently using hospitals. Hospitals that never used glucocorticoids during the acute phase before and after the period were considered as never using hospitals. We considered hospitals that never used glucocorticoids during the acute phase before and started using glucocorticoids after the period as started using hospitals. Hospitals that used glucocorticoids during the acute phase before the period and stopped using glucocorticoids after the period were considered as stopped using hospitals. To avoid the potential misclassification of the four types of hospitals, we included patients who were admitted to hospitals that had at least ten patients with KD per year over the 5-year period. After stratifying the hospitals into four categories, we compared total hospitalization and drug costs, risk of CAL, LOS, total mean dose of IVIG, and use of additional treatment (glucocorticoid use more than 3 days after initial IVIG use, cyclosporine A, infliximab, ulinastatin, plasmapheresis) before January 2012 and after March 2013 using mixed effects models. We also used patient characteristics as fixed effects and hospitals as random effects to account for random variations between the hospitals.

The differences in outcomes of interest were estimated with 95\% confidence intervals (95\%CIs). For the mixed effects linear regression models, log-transformation was required to ensure the error term normality assumption. As the doses of IVIG were missing for 2,345 patients, the analyses relevant to IVIG dose were conducted with the remaining 22,172 patients. We set statistical significance at two-sided $P<0.05$ for all of the analyses. We used STATA software version 14.1 for all data analyses (StataCorp LP, TX, USA).

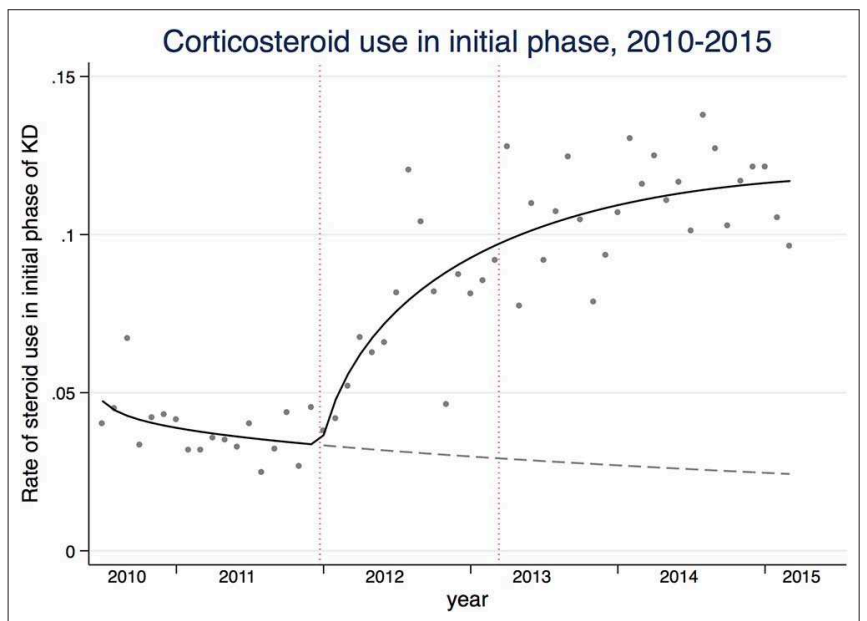

FIGURE 1 | The solid line represents the rates of glucocorticoid use in the acute phase of Kawasaki disease. The gray dashed line represents the counterfactual that would have been observed if the trend were the same before study reports of the efficacy of glucocorticoids and subsequent guideline change. The dashed vertical lines are 3 months before the official publications of the studies (January, 2012) and 3 months after the guideline change (March, 2013)

\section{RESULTS}

We observed a total of 24,517 children hospitalized with KD over the study period. Table 1 shows the summary of the baseline patient characteristics, treatment patterns, and healthcare utilizations, stratified by fiscal years of admissions. In the mixed effects model analyses, glucocorticoid use for both initial and all phases of KD showed an upward trend $\left(P_{\text {trend }}\right.$ $<0.001)$. The spline model showed an increasing trend in glucocorticoid use during the initial phase that started in January 2012 and leveled off after March 2013 (Figure 1). An upward trend in infliximab use was observed $\left(P_{\text {trend }}<0.001\right)$, whereas little changes were observed in dose of IVIG, use of cyclosporine A, ulinastatin, and plasmapheresis. Diagnoses of CALs defined by both ICD-10 and antithrombotic therapy decreased from 4.5 and $1.0 \%$ in 2010 to 2.4 and $0.7 \%$ in 2014 , respectively. Total hospitalization costs and LOS also showed decreasing trends over the study period $\left(P_{\text {trend }}<0.001\right)$.

We identified 91 hospitals that had at least 10 patients per year during 2010-2014, which included 38 consistently using hospitals, 17 started using hospitals, seven stopped using hospitals, and 29 never using hospitals (Table 2). Table 3 shows the total hospitalization costs and risks of CAL stratified by hospital types based on glucocorticoid use in the initial phase of KD. Total hospitalization costs comparing before January 2012 to after 2013 March were reduced in the always using hospital, stopped using hospital, and never using hospitals. The most prominent reduction was observed in started using hospitals (adjusted difference, $-20,295$ JPY; 95\%CI, $-28,865$ to $-13,448)$. In contrast, only stopped using hospitals did not show reduction of hospitalization costs (adjusted difference, $-10,198$ JPY; 95\%CI, $-15,731$ to $-4,479)$. The odds of CAL development were decreased in always using and never using hospitals. The most prominent reduction in odds of CAL was observed in started using hospitals (adjusted OR, 0.22; 95\%CI, 0.07-0.68).

Table 4 shows the changes in total drug costs, LOS, IVIG dose, and additional treatment use. Reduction in total drug costs were observed in the always using, started using, and never using hospitals, whereas an elevated drug cost was found in stopped using hospitals. All hospitals showed decreases in LOS, and the difference was substantial on stopped using hospitals. Only stopped using hospitals showed an increase in average total IVIG dose (adjusted difference, $0.18 \mathrm{mg} / \mathrm{kg}$; 95\%CI, 0.01-0.36).

\section{DISCUSSION}

In the present study, we observed increasing trends in initial phase glucocorticoid use for KD patients, after publications that proved the efficacy of glucocorticoid use for preventing CAL and subsequent KD treatment guideline change. We observed the reduction in hospitalization costs among hospitals that consistently used glucocorticoids, started using after the change in guideline, and never used glucocorticoids. On the other hand, hospitals that stopped using glucocorticoids did not show decreasing trends in hospitalization costs. To our best knowledge, this is the first study that assessed the clinical practice patterns for 
TABLE 1 | Baseline characteristics of children hospitalized with KD by fiscal year of admission and trend analyses for hospital utilizations and adjunctive treatment.

\begin{tabular}{|c|c|c|c|c|c|c|c|}
\hline & \multirow[b]{2}{*}{ Total } & \multicolumn{5}{|c|}{ Fiscal year of admission } & \\
\hline & & 2010 & 2011 & 2012 & 2013 & 2014 & \\
\hline Total Admissions, $n$ & 24,517 & 3,369 & 4,699 & 4,700 & 4,893 & 6,856 & \\
\hline Age (years), mean (SD) & $2.53(2.0)$ & 2.45 (3.9) & $2.46(4.0)$ & $2.48(4.1)$ & $2.55(4.1)$ & $2.65(4.2)$ & \\
\hline Male, $n(\%)$ & $14,146(57.7)$ & 1,980 (58.8) & $2,724(58.0)$ & $2,690(57.2)$ & 2,806 (57.3) & 3,946 (57.6) & \\
\hline Ambulance, $n(\%)$ & $508(2.1)$ & $63(1.9)$ & $74(1.6)$ & $86(1.8)$ & $145(3.0)$ & $140(2.0)$ & \\
\hline Academic, $n(\%)$ & 4,283 (17.5) & 588 (17.5) & $738(15.7)$ & $835(17.8)$ & $997(20.4)$ & $1,125(16.4)$ & \\
\hline \multicolumn{8}{|l|}{ Season } \\
\hline Spring, $n(\%)$ & $5,747(23.4)$ & $400(11.9)$ & $1,237(26.3)$ & 1,234 (26.3) & $1,324(27.1)$ & 1,552 (22.6) & \\
\hline Summer, $n(\%)$ & 6,255 (25.5) & $839(24.9)$ & $1,196(25.5)$ & 1,222 (26.0) & $1,249(25.5)$ & $1,749(25.5)$ & \\
\hline Fall, $n(\%)$ & $5,473(22.3)$ & 907 (26.9) & 981 (20.9) & $999(21.3)$ & 1,020 (20.8) & $1,566(22.8)$ & \\
\hline \multirow[t]{2}{*}{ Winter, $n(\%)$} & $7,042(28.7)$ & 1,223(36.3) & $1,285(27.3)$ & 1,245 (26.5) & 1,300 (26.6) & 1,989 (29.0) & \\
\hline & Total & 2010 & 2011 & 2012 & 2013 & 2014 & $P_{\text {trend }}$ \\
\hline IVIG (g/kg), mean (log-SD) & $2.32(0.01)$ & $2.27(0.01)$ & $2.23(0.01)$ & $2.37(0.01)$ & $2.32(0.01)$ & $2.32(0.01)$ & 0.37 \\
\hline \multicolumn{8}{|l|}{ Glucocorticoid use } \\
\hline All phases, $n(\%)$ & $3,410(13.9)$ & 299 (8.9) & 405 (8.6) & $647(13.8)$ & 869 (17.8) & $1,190(17.4)$ & $<0.001$ \\
\hline Initial phase, $n(\%)$ & 2,007 (8.2) & $141(4.2)$ & $175(3.7)$ & $381(8.1)$ & $516(10.5)$ & $794(11.6)$ & $<0.001$ \\
\hline \multicolumn{8}{|l|}{ Other Treatment } \\
\hline Cyclosporine A, n (\%) & $223(0.9)$ & $23(0.7)$ & $29(0.6)$ & $48(1.0)$ & 59 (1.2) & $64(0.9)$ & 0.11 \\
\hline Infliximab, $n(\%)$ & $105(0.5)$ & $5(0.1)$ & $13(0.2)$ & $8(0.2)$ & $27(0.6)$ & $52(0.8)$ & $<0.001$ \\
\hline Ulinastatin, $n(\%)$ & 955 (3.9) & $83(2.5)$ & $163(3.5)$ & $202(4.3)$ & $227(4.6)$ & $280(4.1)$ & 0.12 \\
\hline Plasmapheresis, $n$ (\%) & $97(0.4)$ & $6(0.2)$ & $11(0.2)$ & $19(0.4)$ & $30(0.6)$ & $31(0.5)$ & 0.17 \\
\hline Intensive care, $n(\%)$ & $181(0.7)$ & $18(0.5)$ & $29(0.6)$ & $34(0.7)$ & $50(1.0)$ & $50(0.7)$ & 0.59 \\
\hline \multicolumn{8}{|l|}{ Outcomes } \\
\hline Readmission, $n$ (\%) & 397 (1.6) & $45(1.3)$ & $79(1.7)$ & $70(1.5)$ & $88(1.8)$ & $115(1.7)$ & 0.62 \\
\hline CAL (ICD-10), $n(\%)$ & 725 (3.0) & $153(4.5)$ & $142(3.0)$ & $137(2.9)$ & $128(2.6)$ & $165(2.4)$ & $<0.001$ \\
\hline CAL (drug), $n(\%)$ & $178(0.7)$ & $34(1.0)$ & $22(0.5)$ & $30(0.6)$ & $44(0.9)$ & $48(0.7)$ & 0.043 \\
\hline Cost, JPY & 300,619 & 314,387 & 310,060 & 302,964 & 298,179 & 288,056 & $<0.001$ \\
\hline LOS, days & 10.8 & 11.0 & 10.8 & 10.8 & 10.8 & 10.3 & $<0.001$ \\
\hline
\end{tabular}

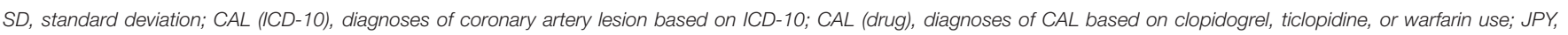
Japanese yen; LOS, length of stay.

TABLE 2 | Selection and timing of steroid use at hospital levels.

\begin{tabular}{|c|c|c|c|}
\hline & \multicolumn{3}{|c|}{ Before January 2012, hospital (\%) } \\
\hline & Non-initial use or never use & Initial use & Tota \\
\hline & 46 & 45 & 91 \\
\hline \multicolumn{4}{|c|}{ After March 2013, hospital (\%) } \\
\hline $\begin{array}{l}\text { Non-initial use or } \\
\text { never use }\end{array}$ & $\begin{array}{c}29 \\
(31.9)\end{array}$ & $\begin{array}{c}7 \\
(7.7)\end{array}$ & 36 \\
\hline Initial use & $\begin{array}{c}17 \\
(18.7)\end{array}$ & $\begin{array}{c}38 \\
(41.8)\end{array}$ & 55 \\
\hline
\end{tabular}

$\mathrm{KD}$ and the impacts of $\mathrm{KD}$ guideline change on risks of CAL and healthcare costs at hospital levels.

Recommendations, regarding to adjunctive therapy for primary treatment with IVIG and ASA and additional therapy in the IVIG-resistant case, are slightly different between the clinical guideline in Japan and that in the US $(2,5)$. First, the risk stratification scores have been proven useful to identify Japanese children in Japan with $\operatorname{KD}(10,11)$, whereas the scores showed low sensitivity and poor negative predictive value outside of Japan (20). Second, recommendations of pulsed methylprednisolone for adjunctive therapy for primary treatment are different between the guidelines in Japan and the US (2, 5). Third, the clinical guideline in Japan describes ulinastatin and plasmapheresis as one of treatment options for additional treatment in the IVIV-resistant cases (2).

An increasing trend in glucocorticoid use was observed over the study period. The trend could be explained by the impacts of previous randomized controlled studies that identified efficacy of glucocorticoids in initial treatment of $\mathrm{KD}$ and subsequent change in Japanese KD guideline during 2012 (4, 15, 16). In fact, our study showed that the upward trend started 3 months before publishing the previous studies and leveled off after change in Japanese $\mathrm{KD}$ guideline. Correspondingly, the risks of CALs showed a decreasing trend. However, we believe that the decreasing trend in CALs could be multifactorial such as upward trends in glucocorticoid use and other aggressive anti-inflammatory treatment, and earlier administrations of IVIG. Indeed, a nationwide survey of $\mathrm{KD}$ in Japan reported that the proportions of IVIG administrations within 5 days 
TABLE 3 | Differences in total costs and proportions of CAL between before 12/2011 and after 4/2013 stratified by use of steroid use defined at hospital levels.

\begin{tabular}{|c|c|c|c|c|}
\hline Hospital characteristics & Always use & Started use & Stopped use & Never use \\
\hline Number of patients, $N$ & 4,029 & 1,544 & 624 & 2,483 \\
\hline Number of hospitals, $N$ & 38 & 17 & 7 & 29 \\
\hline \multicolumn{5}{|l|}{ Total hospitalization cost } \\
\hline Before 12/2011, mean JPY (log-SD) & $\begin{array}{c}301,374 \\
(0.5)\end{array}$ & $\begin{array}{c}289,294 \\
(0.5)\end{array}$ & $\begin{array}{c}304,882 \\
(0.6)\end{array}$ & $\begin{array}{c}286,081 \\
(0.6)\end{array}$ \\
\hline After 4/2013, mean JPY (log-SD) & $\begin{array}{c}290,259 \\
(0.5)\end{array}$ & $\begin{array}{c}266,495 \\
(0.5)\end{array}$ & $\begin{array}{l}321,142 \\
(0.6)\end{array}$ & $\begin{array}{c}279,568 \\
(0.5)\end{array}$ \\
\hline Adjusted Difference, JPY (95\%Cl) & $\begin{array}{c}-11,270 \\
(-15,546,-6,887)\end{array}$ & $\begin{array}{c}-20,295 \\
(-26,865,-13,448)\end{array}$ & $\begin{array}{c}7,926 \\
(-5,838,22,856)\end{array}$ & $\begin{array}{c}-10,198 \\
(-15,731,-4,479)\end{array}$ \\
\hline \multicolumn{5}{|l|}{ CAL } \\
\hline Before 12/2011, N (\%) & $\begin{array}{c}59 \\
(3.5)\end{array}$ & $\begin{array}{c}11 \\
(1.9)\end{array}$ & $\begin{array}{c}11 \\
(3.0)\end{array}$ & $\begin{array}{c}33 \\
(3.2)\end{array}$ \\
\hline After 4/2013, N (\%) & $\begin{array}{c}44 \\
(1.9)\end{array}$ & $\begin{array}{c}5 \\
(0.5)\end{array}$ & $\begin{array}{c}4 \\
(1.5)\end{array}$ & $\begin{array}{c}27 \\
(1.9)\end{array}$ \\
\hline Adjusted OR (95\%Cl) & $\begin{array}{c}0.54 \\
(0.35,0.82)\end{array}$ & $\begin{array}{c}0.22 \\
(0.07,0.68)\end{array}$ & $\begin{array}{c}0.52 \\
(0.15,1.77)\end{array}$ & $\begin{array}{c}0.50 \\
(0.29,0.86)\end{array}$ \\
\hline
\end{tabular}

JPY, Japanese-Yen; Log-SD, log-transformed standard deviation; CAL, coronary artery lesion; Cl, confidence interval; OR, odds ratio.

TABLE 4 | Differences in drug costs, mean intravenous immunoglobulin (IVIG) dose, length of hospital stay (LOS), proportions of additional treatment between before $12 / 2011$ and after 4/2013 stratified by use of steroid use defined at hospital levels.

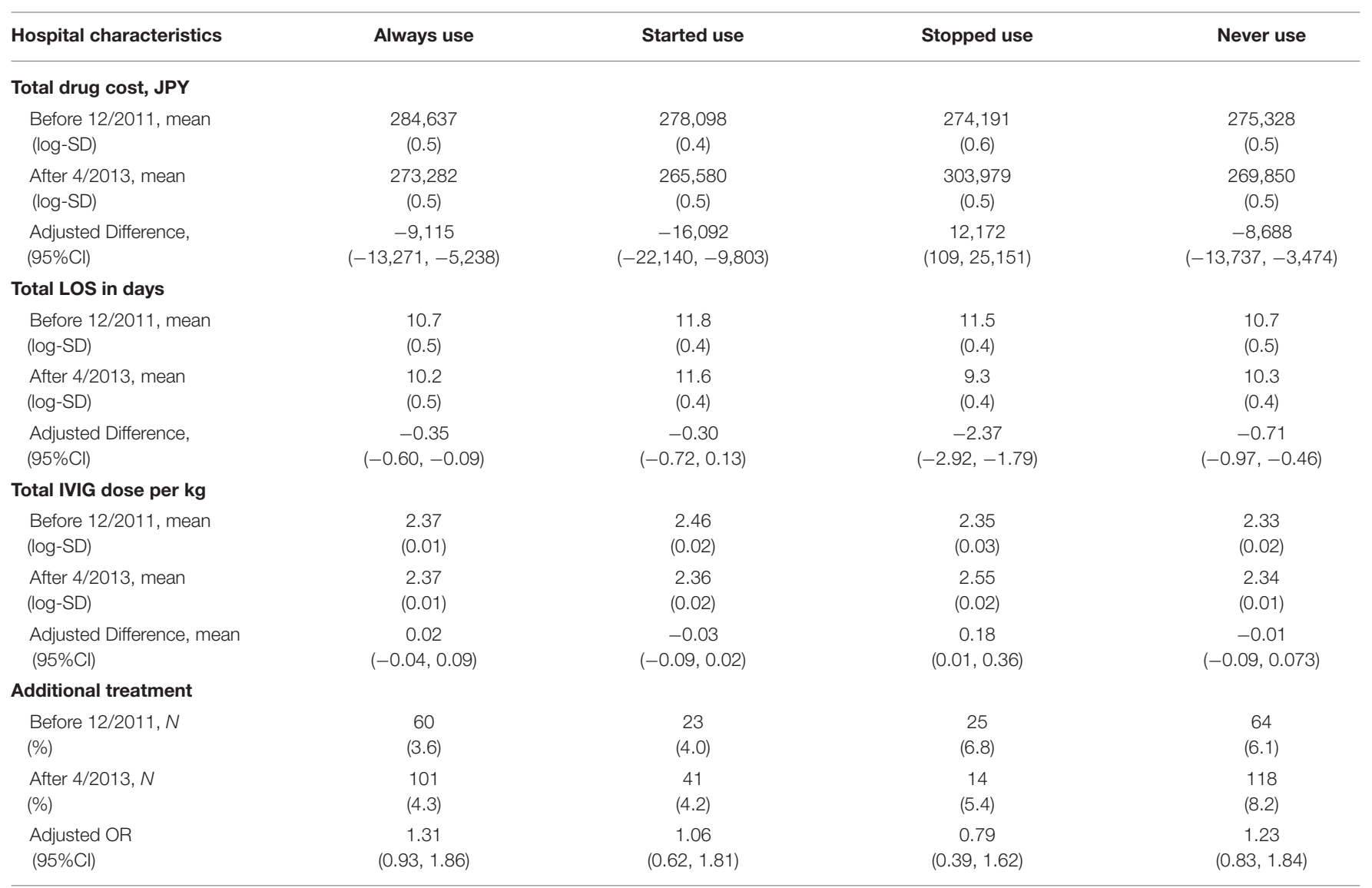

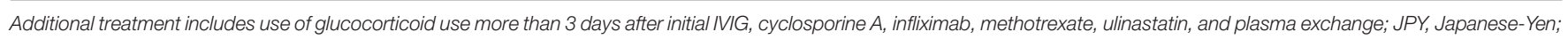
Log-SD, log-transformed standard deviation; CAL, coronary artery lesion; Cl, confidence interval; OR, odds ratio. 
increased from $67.7 \%$ in 2010 to $69.2 \%$ in 2014 (4). Furthermore, slightly upward trends in use of infliximab and ulinastatin were observed in our study, probably because of the guideline recommendations for early aggressive anti-inflammatory therapy and the study results that reported the effectiveness of infliximab and ulinastatin $(4,21-24)$. We believe that these factors may have contributed to the change in treatment strategy and reduction in risks of CAL, rather than a single effect of glucocorticoid use in the initial phase.

The effects of changes in treatment strategies at hospital levels on the risk of CAL and healthcare costs are uncertain, and finding a cost-saving strategy with improving prognosis is extremely important considering the increasing healthcare expenditure in high-income countries (25). We observed decreasing trends in risks of CAL and healthcare costs among hospitals that used glucocorticoids consistently and those that never used them. As we cited above, we believe that the decreasing trends in the risks of CAL reflected several factors such as increasing use of glucocorticoid, earlier administrations, and elevated doses of IVIG. Furthermore, decreasing trends in healthcare costs in consistently and never using hospitals could be explained by natural trends in healthcare costs among pediatric inpatients in Japan, rather than effects of change in the clinical guideline for KD. In fact, downward trends in total hospitalization costs were already observed for other pediatric disorders in Japan, such as immune thrombocytopenia and respiratory infections (26-28).

Complying with the changes in guideline in terms of glucocorticoid use in the initial phase of KD could be the most effective strategy to save healthcare costs for $\mathrm{KD}$ inpatients. Indeed, we observed the greatest reduction in healthcare costs (-20,295 JPY per KD inpatients) among hospitals that started using glucocorticoids compared with consistently using and never using hospitals. The greatest reduction in total hospitalization costs in the started using hospitals could reflect decreases in drug costs and slightly decreased LOS. On the other hand, stopped using hospitals showed slightly elevated healthcare costs, which contradicted to natural trends in reduction of healthcare costs. Considering for the increase in IVIG dose, we believe that the stopping the use of initial phase glucocorticoid use may have increased the cases of non-response to initial IVIG, which resulted in elevated total IVIG dose and subsequent increases in hospitalization costs.

We acknowledge several limitations to our study. The number of $\mathrm{KD}$ inpatients and subsequent development of CAL could have been underestimated because of possible underreporting and/or misclassification of ICD-10 code. However, we maximized diagnostic accuracy of $\mathrm{KD}$ and $\mathrm{CAL}$ by restricting to patients

\section{REFERENCES}

1. Kawasaki T, Kosaki F, Okawa S, Shigematsu I, Yanagawa H. A new infantile acute febrile mucocutaneous lymph node syndrome prevailing in Japan. Pediatrics. (1974) 54:272-6.

2. Research Committee of the Japanese Society of Pediatric Cardiology; Cardiac Surgery Committee for Development of Guidelines for Medical Treatment of Acute Kawasaki Disease. Guidelines for medical treatment of acute Kawasaki disease: report of the research committee of the japanese society of pediatric who received IVIG and identified patients with antithrombotic treatment. As a result, our estimates of CAL were mostly similar to the findings from the previously reported national survey in Japan. The detailed clinical presentation, laboratory data, and patient information for the follow-up of $\mathrm{KD}$ at outpatient visits were unavailable in the DPC database. Due to these limitations, we were unable to reliably evaluate and compare the difference of pre-treatment severity scores among KD patients, and we could not compare patients between hospitals with different treatment strategies. In addition, we were not able to detect cases of CAL in $\mathrm{KD}$ patients that were detected during outpatient follow-up periods. The strength of this study was the use of the DPC database, which is the only national inpatient database currently available in Japan. Using the DPC database, we calculated the robust estimates of the recent clinical practice patterns in $\mathrm{KD}$ and the impact of change in $\mathrm{KD}$ guideline at hospital levels throughout Japan.

In summary, this study added novel insights into strategies of KD treatment by comparing the different treatment strategies. Glucocorticoid treatment in the initial phase of KD could be a cost-saving strategy with improving clinical outcomes. We believe that our investigations showed valuable information to improve prognosis for CAL and save healthcare expenditure at hospital levels.

\section{DATA AVAILABILITY STATEMENT}

The raw data supporting the conclusions of this article will be made available by the authors, without undue reservation, to any qualified researcher.

\section{AUTHOR CONTRIBUTIONS}

YO and NMi designed the data collection instruments, coordinated data, drafted the initial manuscript, and performed the initial analyses. KF, HY, MM, TK, HM, and NMo supervised data collection, revised the manuscript, and approved the final manuscript as submitted. Each author listed on the manuscript has seen and approved the submission of this version of the manuscript and takes full responsibility for its contents.

\section{FUNDING}

This work was supported by grants from Ministry of Health, Labour, and Welfare, Japan, and the Japan Agency for Medical Research and Development. cardiology and cardiac surgery (2012 revised version). Pediatr Int. (2014) 56:135-58. doi: 10.1111/ped.12317

3. Okubo Y, Nochioka K, Sakakibara H, Testa M, Sundel RP. National survey of pediatric hospitalizations due to Kawasaki disease and coronary artery aneurysms in the USA. Clin Rheumatol. (2017) 36:413-9. doi: $10.1007 /$ s10067-016-3512-6

4. The Nationwide Survey of Kawasaki Disease (Japanese). Available online at: http://www.jichi.ac.jp/dph/kawasaki.html (accessed January 30, 2018). 
5. McCrindle BW, Rowley AH, Newburger JW, Burns JC, Bolger AF, Gewitz M, et al. Diagnosis, treatment, and long-term management of Kawasaki disease. Circulation. (2017) 135:e927-99. doi: 10.1161/CIR.0000000000000484

6. Terai M, Shulman ST. Prevalence of coronary artery abnormalities in Kawasaki disease is highly dependent on gamma globulin dose, but independent of salicylate dose. J Pediatr. (1997) 131:888-93. doi: 10.1016/S0022-3476(97)70038-6

7. Durongpisitkul K, Gururaj VJ, Park JM, Martin CF. The prevention of coronary artery aneurysm in Kawasaki disease: a meta-analysis on the efficacy of aspirin ad immune globulin treatment. Pediatrics. (1995) 96:1057-61.

8. Burns JC, Capparelli EV, Brown JA, Newburger JW, Glode MP. Intravenous gamma-globulin treatment and retreatment in Kawasaki disease. US/Canadian Kawasaki Syndrome Study Group. Pediatr Infect Dis J. (1998) 17:1144-8. doi: 10.1097/00006454-199812000-00009

9. Tremoulet AH, Best BM, Song S, Wang S, Corinaldesi E, Eichenfield JR, et al. Resistance to intravenous immunoglobulin in children with Kawasaki Disease. J Pediatr. (2008) 153:117-21. doi: 10.1016/j.jpeds.2007.12.021

10. Kobayashi T, Inoue Y, Otani Y, Morikawa A, Takeuchi K, Saji T, et al. Risk stratification in the decision to include prednisolone with intravenous immunoglobulin in primary therapy of Kawasaki disease. Pediatr Infect Dis J. (2009) 28:498-502. doi: 10.1097/INF.0b013e3181950b64

11. Kobayashi T, Inoue Y, Takeuchi K, Okada Y, Tamura K, Tomomasa $\mathrm{T}$, et al. Prediction of intravenous immunoglobulin unresponsiveness in patients with Kawasaki disease. Circulation. (2006) 113:2606-12. doi: 10.1161/CIRCULATIONAHA.105.592865

12. Egami K, Muta H, Ishii M, Suda K, Sugahara $Y$, Ienuma $M$, et al. Prediction of resistance to intravenous immunoglobulin treatment in patients with Kawasaki disease. J Pediatr. (2006) 149:237-40. doi: 10.1016/j.jpeds. 2006.03.050

13. Sano T, Kurotobi S, Matsuzaki K, Yamamoto T, Maki I, Miki K, et al. Prediction of non-responsiveness to standard high-dose gamma-globulin therapy in patients with acute Kawasaki disease before starting initial treatment. Eur J Pediatr. (2007) 166:131-7. doi: 10.1007/s00431-006-0223-Z

14. Okubo Y, Michihata N, Morisaki N, Sundel RP, Matsui H, Fushimi K, et al. Association between dose of glucocorticoids and coronary artery lesion in Kawasaki disease. Arthritis Care Res. (2018) 70:1052-7. doi: 10.1002/acr.23456

15. Ogata S, Ogihara Y, Honda T, Kon S, Akiyama K, Ishii M. Corticosteroid pulse combination therapy for refractory Kawasaki disease: a randomized trial. Pediatrics. (2012) 129:e17-23. doi: 10.1542/peds.2011-0148

16. Kobayashi T, Saji T, Otani T, Takeuchi K, Nakamura T, Arakawa H, et al. Efficacy of immunoglobulin plus prednisolone for prevention of coronary artery abnormalities in severe Kawasaki disease (RAISE study): a randomized, open-label, blinded-endpoints trial. Lancet. (2012) 379:1613-20. doi: 10.1016/S0140-6736(11)61930-2

17. Matsuda S, Fujimori K, Kuwabara K, Ishikawa KB, Fushimi K. Diagnosis procedure combination as an infrastructure for the clinical study. Asian Pac J Dis Manage. (2011) 5:81-7. doi: 10.7223/apjdm.5.81

18. The World Health Organization Child Growth Standards. Available online at: http://www.who.int/childgrowth/en/ (accessed April 15, 2017).

19. Wagner AK, Soumerai SB, Zhang F, Ross-Degnan D. Segmented regression analysis of interrupted time series studies in medication use research.
J Clin Pharm Ther. (2002) 27:299-309. doi: 10.1046/j.1365-2710.2002. 00430.x

20. Sleeper LA, Minich LL, McCrindle BM, Li JS, Colan SD, et al. Pediatric Heart Network Investigators. Evaluation of Kawasaki Disease risk-scoring systems for intravenous immunoglobulin resistance. J Pediatr. (2011) 158:831-5.e3. doi: $10.1016 /$ j.jpeds.2010.10.031

21. Rodriguez-Gonzalez M, Matamala-Morillo MA, Segado-Arenas A. Infliximab as rescue therapy in refrectory Kawasaki disease. Ann Pediatr Cardiol. (2014) 7:74-5. doi: 10.4103/0974-2069.126578

22. Masuda H, Kobayashi T, Hachiya A, Nakashima Y, Shimizu H, Nozawa T, et al. Inflixmab for the treatment of refractory Kawasaki Disease: A Nationwide Survey in Japan. J Pediatr. (2018) 195:115-20. doi: 10.1016/j.jpeds.2017. 10.013

23. Kanai $T$, Ishiwata $T$, Kobayashi $T$, Sato $H$, Takizawa M,Kawamura $Y$, Tsujimoto $\mathrm{H}$, et al. Ulinastatin, a urinary trypsin inhibitor, for the initial treatment of patients with Kawasaki disease: a retrospective study. Circulation. (2011) 124:2822-8. doi: 10.1161/CIRCULATIONAHA.111. 028423

24. Iwashima S, Seguchi M, Matubayashi T, Ohzeki T, Ulinastatin therapy in Kawasaki disease. Clin Drug Investig. (2007) 27:691-6. doi: 10.2165/00044011-200727100-00004

25. Papanicolas I, Woskie LR, Jha AK. Health care spending in the United States and other high-income countries. JAMA. (2018) 319:1024-39. doi: 10.1001/jama.2018.1150

26. Okubo Y, Michihata N, Morisaki N, Uda K, Miyairi I, Ogawa Y, et al. Recent trends in practice patterns and impact of corticosteroid use on pediatric Mycoplasma pneumoniae-related respiratory infections. Respir Investig. (2018) 56:158-65. doi: 10.1016/j.resinv.2017.11.005

27. Okubo Y, Michihata N, Morisaki N, Hangai M, Matsui G, Fushimi K, et al. Recent trends in practice patterns and comparisons between immunoglobulin and corticosteroid in pediatric immune thrombocytopenia. Int J Hematol. (2018) 107:75-82. doi: 10.1007/s12185-017-2322-1

28. Uda K, Okubo Y, Shoji K, Miyairi I, Morisaki N, Michihata N, et al. Trends of neuraminidase inhibitors use in children with influenza related respiratory infections. Pediatr Pulmonol. (2018) 53:802-8. doi: 10.1002/ppul.24021

Conflict of Interest: MM and TK received honorariums from the Japan Blood Products Organization and Teijin Pharma Limited Nihon Pharmaceutical Co. MM received an honorarium from the Mitsubishi Tanabe Pharma Corporation.

The remaining authors declare that the research was conducted in the absence of any commercial or financial relationships that could be construed as a potential conflict of interest.

Copyright (C) 2020 Okubo, Miura, Kobayashi, Morisaki, Michihata, Matsui, Fushimi and Yasunaga. This is an open-access article distributed under the terms of the Creative Commons Attribution License (CC BY). The use, distribution or reproduction in other forums is permitted, provided the original author(s) and the copyright owner(s) are credited and that the original publication in this journal is cited, in accordance with accepted academic practice. No use, distribution or reproduction is permitted which does not comply with these terms. 